\title{
Sofosbuvir and daclatasvir are safe and effective in treatment of recurrent hepatitis $C$ virus in Egyptian patients underwent living donor liver transplantation
}

Osama Aboelfotoh Aly ${ }^{1}$, Wael Ahmed Yousry ${ }^{1}$, Nahla Mohamed Teama ${ }^{2^{*}}$ (D), Eman Mohammed Shona ${ }^{1}$ and Ahmed Mohamed ElGhandour ${ }^{1}$

\begin{abstract}
Background: Liver transplant population has been considered as a special population in the treatment of hepatitis C virus infection, not only because of lower sustained virological response (SVR) rates in comparison with pretransplant setting, but also for other aspects (i.e., immunosuppressive therapy, renal function, drug-drug interactions). We aimed to evaluate the efficacy and safety of the combined treatment with sofosbuvir and daclatasvir with or without ribavirin in liver transplant recipients with recurrent hepatitis $\mathrm{C}$ following transplantation and screening for the development of hepatocellular carcinoma during treatment, after the end of treatment, or during follow-up. This multicenteric prospective study was conducted in Egypt. This study included 40 patients who underwent living donor liver transplantation that started treatment at least 3 months following transplantation. All participants received $400 \mathrm{mg}$ sofosbuvir once daily plus daclatasvir $60 \mathrm{mg}$ daily \pm ribavirin. Treatment lasted for up to 24 weeks, and participants were followed up as outpatients monthly for 12 and 24 weeks and 36 weeks post-treatment to determine sustained virological response (SVR12 and SVR24), considered to be a cure and detection of any changes in tumor markers or radiological imaging during follow-up.
\end{abstract}

Results: In the current study, 40 patients (100\%) have good response to treatment during treatment and during followup (SVR 12 was 100\%). No abnormal side effects to treatment were detected; also, no drug-drug interactions were noted during the treatment.

Conclusions: Treatment of HCV after living donor liver transplantation with combined sofosbuvir and daclatasvir is safe and well-tolerated and provides high rates of SVR.

Keywords: Hepatitis C virus infection, Sofosbuvir, Daclatasvir, Living donor liver transplantation

\section{Background}

End-stage liver disease (ESLD) caused by hepatitis $C$ virus $(\mathrm{HCV})$ infection is the main indication of liver transplantation (LT) in most transplant programs [1].

Most patients will go on to develop viral recurrence, and it has been widely appreciated that the clinical course of $\mathrm{HCV}$ in $\mathrm{LT}$ recipients is accelerated in comparison with

\footnotetext{
* Correspondence: nahlateama@med.asu.edu.eg

${ }^{2}$ Division of Nephrology, Department of Internal Medicine, Faculty of

Medicine, Ain Shams University, Ramsis street 38, Abbasia, Cairo 11566, Egypt Full list of author information is available at the end of the article
}

chronic hepatitis $\mathrm{C}$ in the non-transplant population, such that 8 to $44 \%$ of the patients will go on to develop cirrhosis within 5 to 10 years after transplantation [2].

Clinical decompensation in a significant proportion of $\mathrm{HCV}$ recipients who have developed cirrhosis of the allograft, with $40 \%$ developing clinical decompensation at 1 year, and up to $60 \%$ will suffer a decompensation episode 3 years after the diagnosis of cirrhosis. This unfortunately significantly affects patient survival, with an estimated survival rate of less than $10 \%$ at 3 years [3]. 
Treatment of hepatitis $\mathrm{C}$ in the post-liver transplantation patient is a rapidly evolving field. When treating hepatitis $C$ in this setting, the main goals of therapy include (a) cure of HCV chronic infection in the allograft post-transplant; (b) minimize the risk of developing $\mathrm{HCV}$-associated complications in the allograft, such as fibrosing cholestatic hepatitis and allograft failure; and (c) prevent the development of hepatic fibrosis and thus preserve the function of the transplanted liver [4].

Treatment with pegylated interferon (PEG-IFN) and ribavirin (RBV) had been the standard of care for $\mathrm{HCV}$ patients for a decade, until the development of several direct-acting antivirals (DAAs) [5].

The association of sofosbuvir and daclatasvir has been shown to have very high antiviral efficacy when administered with or without ribavirin to previously naïve or non-responder patients with chronic $\mathrm{HCV}$ infection. Combination with daclatasvir in a LT recipient with severe recurrent cholestatic hepatitis $\mathrm{C}$ has been reported, showing a favorable outcome and the lack of drug interactions with calcineurin inhibitors (CNI) [6].

Owing to the low incidence of HCC among SVR patients, the study of factors associated with liver cancer development in this population is rather problematic. So far, no single clinical or histological predictor of HCC development has been identified in SVR patients, reinforcing therefore the concept that $\mathrm{HCC}$ risk in $\mathrm{HCV}$ patients may be multifactorial [7].

The aim of this study was to evaluate the virological response, clinical efficacy, and safety of the combined sofosbuvir and daclatasvir in living donor liver transplant recipients with recurrent hepatitis $\mathrm{C}$ following transplantation and screening for the development of hepatocellular carcinoma during treatment, after the end of treatment, or during follow-up.

\section{Methods}

This was a multicenteric prospective study conducted in Egypt. This study included 40 patients who underwent living donor liver transplantation who started treatment at least 3 months following transplantation provided that no abnormality was detected in liver functions related to immunosuppression and no prior history of treatment of hepatitis C infection. All patients were fully investigated for complete blood picture, liver function tests, coagulation profile, sodium, potassium, kidney function tests, HCV antibodies and quantitative HCV RNA by PCR, HBV markers, tumor markers including alpha fetoprotein (AFP), and abdominal ultrasonography.

Eligible patients had no evidence of advanced fibrosis (METAVIR score $\leq$ F2) on fibroscan performed not more than 6 months before screening.

All participants received $400 \mathrm{mg}$ sofosbuvir once daily plus daclatasvir $60 \mathrm{mg}$ daily \pm ribavirin. Treatment lasted for up to 24 weeks, and participants were followed up as outpatients monthly for 12 and 24 weeks and 36 weeks post-treatment to determine sustained virological response (SVR12 and SVR24); also, patients were followed up for any changes in tumor markers or radiological imaging during follow-up.

A stable tacrolimus-based or cyclosporine-based immunosuppressive regimen was required, and glucocorticoids were permitted at a dose of $5 \mathrm{mg}$ per day.

\section{Statistical analysis}

All collected data were analyzed and correlated. Statistical analysis was performed using the Statistical Package for Social Sciences (SPSS) version 24. Basic descriptive statistics including means and standard deviations were performed. A comparison of qualitative data between groups was performed using the chi-square test. Independent test or Mann-Whitney $U$ test was used to compare quantitative data between groups with parametric distribution or nonparametric distribution, respectively. The continuous variables across time were compared using the paired $t$ test or Wilcoxon signed-rank test. Differences were considered statistically significant if the $p$ value was less than 0.05 .

\section{Results}

This prospective multicenteric study included 40 patients, 37 males (92.5\%) and 3 females (7.5\%); their mean age was $55.3 \pm 5.6(33-62)$ years. Thirteen of them were diabetics (32.5\%) and 27 (67.5\%) were not diabetics.

There is a statistically significant reduction in hemoglobin levels at the first month after starting treatment $(p=0.013)$ and 9 months after the end of treatment $(p=0.018)$. Otherwise, the reduction at the other different treatment stages and follow-up is not significant.

As regards the platelet count, there is a statistically significant increase at the first month $(p=0.003)$ after onset of the treatment and 9 months after the end of the treatment $(p=0.005)$. There is a highly statistically significant increase in platelet count at 6 months after the end of treatment $(p<0.001)$; otherwise, the increase at the other different treatment stages is insignificant.

There is a statistically significant decrease in ALT levels at the first month after starting treatment $(p=0.001)$ than that before starting treatment and a statistically significant decrease after the end of treatment $(p-0.004)$; also, there is a highly statistically significant decrease in ALT at other different stages of treatment $(p<00.1)$, but there is no statistically significant change in AST levels at different stages of treatment or at follow-up. The same in AFP that shows no statistically significant change in its levels at different stages of treatment or after the end of treatment.

There is a highly statistically significant increase in serum albumin level after the end of the treatment $(p=0.001)$ and a statistically significant increase in serum albumin level at 
3 months after the end of treatment $(p=0.019)$, at 6 months after the end of treatment $(p=0.04)$, and at 9 months after the end of treatment (0.004). As regards ALP, there is a statistically significant decrease in alkaline phosphatase level at 1 month after starting treatment $(p=0.002)$, after the end of treatment $(p=$ $0.002)$, and 6 months after the end of treatment $(p=$ 0.002) than that before starting treatment, while there is a highly statistically significant decrease in alkaline phosphatase levels at 3 months $(p=0.00)$ and at 9 months $(p 0.00)$ after the end of the treatment.

There is a significant decrease in bilirubin levels at the end of treatment $(p=0.003), 6$ months after treatment ( $p=$ $0.04)$, and 9 months after the end of treatment $(p=0.045)$, while there is no significant difference first month after starting treatment and 3 months after the end of treatment.

There is a highly statistically significant decrease in blood urea level at 3 months after the end of treatment $(p=0.001)$.

There is a statistically significant decrease in urea at the end of treatment $(p=0.005), 6$ months after the end of treatment $(p=0.005)$, and 9 months after treatment (0.002), but there is no statistically significant difference in blood urea levels at 1 month after starting treatment; also, there is no statistically significant difference between serum creatinine levels at different stages of treatment and follow-up.

There is a highly statistically significant decrease in INR levels at the first month after starting treatment ( $p$ $=0.00$ ). There is a significant decrease in INR levels at 3 months after the end of treatment $(p=0.002)$.
There is an insignificant decrease in INR levels after the end of treatment and 6 and 9 months after the end of treatment (Table 1).

All cases had negative PCR after the first month of treatment and continued till the end of follow-up (Table 2).

No ultrasonographic changes as regards hepatic focal lesion were detected during the course of treatment or after the course of treatment up to 9 months (Table 3).

Table 4 shows fibroscan results of the patients before treatment.

\section{Discussion}

This study included 40 patients who underwent living donor liver transplantation that started treatment at least 3 months following transplantation and no prior history of treatment of hepatitis $\mathrm{C}$ infection.

This study showed that $100 \%$ of patients achieved SVR after treatment of $\mathrm{HCV}$ with combined sofosbuvir and daclatasvir.

Poordad and his colleagues showed that 12 weeks of treatment with the pan-genotypic combination of daclatasvir with sofosbuvir and ribavirin achieved SVR12 rates of $83 \%$ and $94 \%$ in the advanced cirrhosis and posttransplantation cohorts, respectively [8].

Another study by Pellicelli and his colleagues evaluated the safety and efficacy of sofosbuvir and daclatasvir with or without RBV among 12 post-LT patients with severe recurrent $\mathrm{HCV}$ who were treated through an international compassionate use program. Three patients had fibrosing cholestatic HCV and 9 had cirrhosis. The mean MELD at baseline was 22 and the mean Child-

Table 1 Comparison between baseline and follow-up values of lab investigations of patients

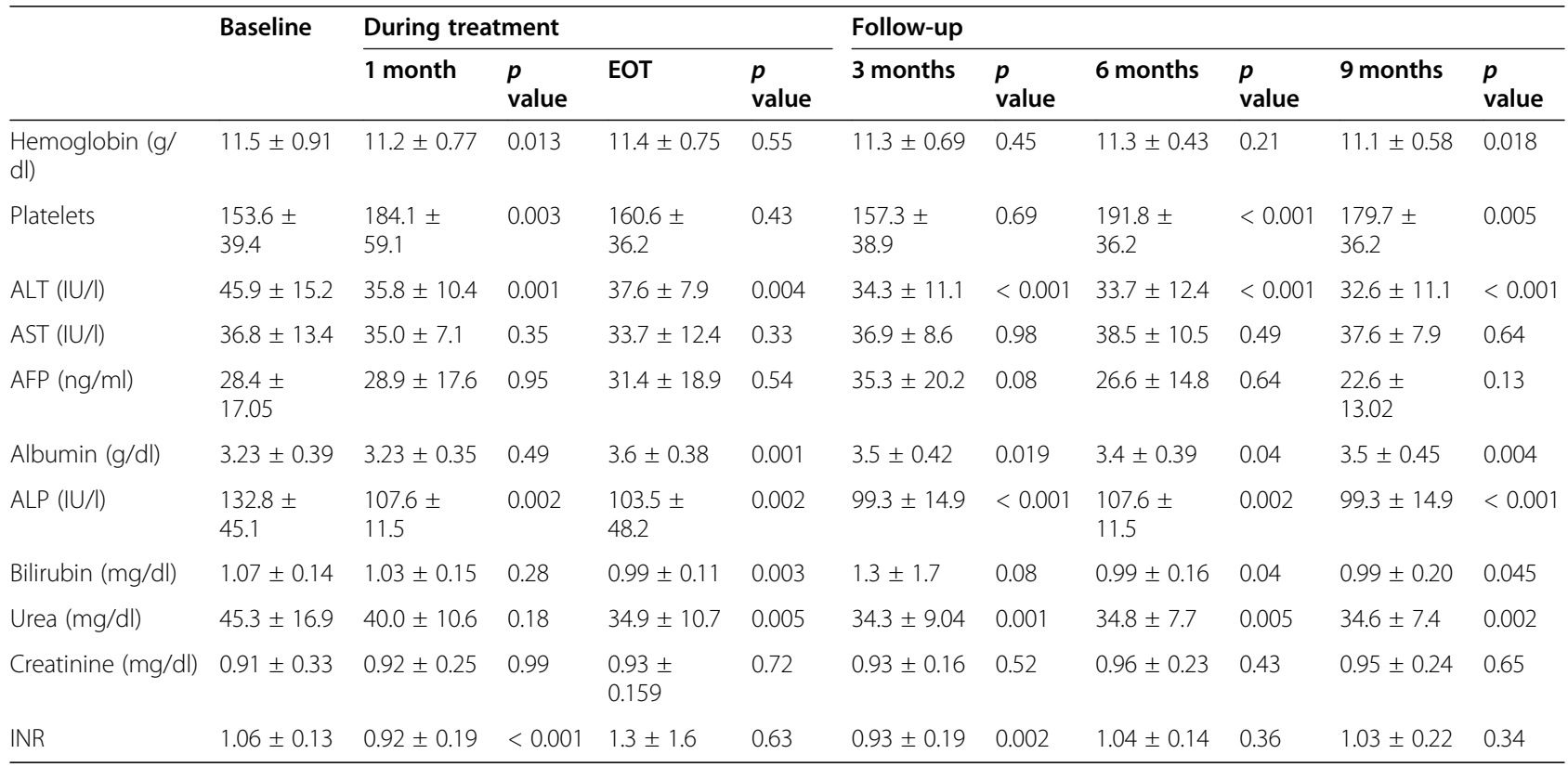

EOT end of treatment, ALT alanine aminotransferase, AST aspartate aminotransferase, AFP alpha fetoprotein, ALP alkaline phosphatase 
Table 2 PCR changes during treatment

\begin{tabular}{|c|c|c|}
\hline Item & Frequency & Percentage \\
\hline \multicolumn{3}{|l|}{ PCR before } \\
\hline Mean \pm SD & $366057.5 \pm 376220.9$ & \\
\hline Min-max & $698-2000000$ & \\
\hline \multicolumn{3}{|c|}{ PCR 1 month after starting treatment } \\
\hline Positive & 0 & 0 \\
\hline Negative & 40 & 100 \\
\hline \multicolumn{3}{|c|}{ PCR after the end of treatment } \\
\hline Positive & 0 & 0 \\
\hline Negative & 40 & 100 \\
\hline \multicolumn{3}{|c|}{ PCR 3 months after the end of treatment } \\
\hline Positive & 0 & 0 \\
\hline Negative & 40 & 100 \\
\hline \multicolumn{3}{|c|}{ PCR 6 months after the end of treatment } \\
\hline Positive & 0 & 0 \\
\hline Negative & 40 & 100 \\
\hline \multicolumn{3}{|c|}{ PCR 9 months after the end of treatment } \\
\hline Positive & 0 & 0 \\
\hline Negative & 40 & 100 \\
\hline
\end{tabular}

Pugh score was 10. During treatment, 3 deaths occurred: one due to rapidly progressive liver failure, one due to gastrointestinal bleeding, and one due to septic shock. Severe adverse events occurred among 4 of the remaining 9 patients and were attributed to the severity Table 3 Ultrasonographic changes during the course of treatment

\begin{tabular}{|c|c|c|}
\hline Item & Frequency & Percentage \\
\hline \multicolumn{3}{|c|}{ US before treatment } \\
\hline Splenomegaly & 6 & 155 \\
\hline Normal & 24 & 85 \\
\hline \multicolumn{3}{|c|}{ US 1 month after starting treatment } \\
\hline HFL & 0 & 0 \\
\hline No HFL & 40 & 100 \\
\hline \multicolumn{3}{|c|}{ US after the end of treatment } \\
\hline HFL & 0 & 0 \\
\hline No HFL & 40 & 100 \\
\hline \multicolumn{3}{|c|}{ US 3 months after the end of treatment } \\
\hline HFL & 0 & 0 \\
\hline No HFL & 40 & 100 \\
\hline \multicolumn{3}{|c|}{ US 6 months after the end of treatment } \\
\hline HFL & 0 & 0 \\
\hline No HFL & 40 & 100 \\
\hline \multicolumn{3}{|c|}{ US 9 months after the end of treatment } \\
\hline HFL & 0 & 0 \\
\hline No HFL & 40 & 100 \\
\hline
\end{tabular}

HFL hepatic focal lesion
Table 4 Fibroscan of the patients at baseline

\begin{tabular}{ll}
\hline Values & $\boldsymbol{N}(\%)$ \\
\hline F1 & $11(27.5)$ \\
F2 & $29(72.5)$ \\
\hline F1 minimal fibrosis, F2 fibrosis has occurred and spread inside the areas of the \\
liver including blood vessels
\end{tabular}

of the patients' underlying liver disease rather than directly to the antiviral treatment. The 9 patients who completed 24 weeks of treatment all had undetectable HCV RNA at the end of treatment. At the time the study was published, post-treatment virologic data was available for 5 patients, all of whom had achieved SVR (24). In this series as well as an additional case report describing the successful treatment of a patient with post-LT fibrosing cholestatic HCV with sofosbuvir and daclatasvir for 24 weeks, there were no apparent drug-drug interactions between DAA and tacrolimus [9].

High rates of virologic and clinical response were seen in 23 patients with post-LT fibrosing cholestatic $\mathrm{HCV}$ treated with sofosbuvir $(n=8)$ or sofosbuvir and daclatasvir $(n=15)$ based regimens [10].

An ongoing prospective multicenter study in France by Manns et al. evaluating the efficacy of sofosbuvir and daclatasvir with or without ribavirin has found high SVR rates in post-LT patients with aggressive recurrent $\mathrm{HCV}$ infection [11].

In a study by Suraweera and his colleagues, they reported data on $130 \mathrm{LT}$ patients who underwent treatment for 12 or 24 weeks. The mean time period between LT and treatment was $74.2( \pm 73.5)$ months. Eleven patients received sofosbuvir and daclatasvir without ribavirin for 12 weeks and had a response rate of $100 \%$ at the end of treatment as well as a SVR12 rate of $100 \%$. Sixty-four patients underwent this same regimen for 24 weeks and had a response rate of $100 \%$ at the end of treatment and a SVR12 rate of $97 \%$. Three patients received sofosbuvir and daclatasvir with ribavirin for 12 weeks; the end-oftreatment response rate was $67 \%$, with a SVR12 rate of $67 \%$. Fifty-two patients underwent the same regimen for 24 weeks and achieved an end-of-treatment response rate of $98 \%$ and a SVR12 rate of $96 \%$ [12].

The authors also concluded that ribavirin did not have a statistically significant influence on SVR and that further prognostic factors needed to be defined. Thirty patients were reported to have experienced severe side effects, with the most common being hematologic toxicity. Two patients died, 1 from diabetic coma and the other from HCV recurrence at 6 weeks post-treatment. Tacrolimus dosing was adjusted in $56 \%$ of patients, cyclosporine in $49 \%$ of patients, and everolimus in $38 \%$ of patients [12].

In the current study, the initial values of bilirubin, alkaline phosphatase, and liver enzymes were normal at baseline and improved during the treatment period and 
follow-up. No liver toxicity of the administered antivirals was observed. The values of albumin and platelets are significantly improved during treatment and follow-up.

Also, there were no changes in the ultrasonographic findings noted after starting treatment and there were no hepatic focal lesions detected during treatment or follow-up period.

The study regimen was compatible with multiple immunosuppressive regimens without dose adjustments, and there were no events of graft rejection.

In contrast, Price et al. found that regimens containing the NS3 protease inhibitors simeprevir or paritaprevir can have significant pharmacokinetic interactions with calcineurin inhibitors [13].

Adverse events during treatment were consistent with the underlying disease; no treatment-specific safety signals were apparent and no serious adverse events were detected due to treatment protocol.

According to data from eight studies being presented at The International Liver Congress ${ }^{\mathrm{TM}} 2017$ in Amsterdam, The Netherlands, there remains continued debate on whether patients are at risk of developing liver cancer after achieving sustained virological response (SVR) with a direct-acting antiviral (DAA) regimen for hepatitis $\mathrm{C}$ virus (HCV).

Despite Trépo and his colleagues found initially promising results, more than one study investigating genetic predisposition to liver cancer failed to identify any robust predictor of HCC development in HCV patients that can be used in clinical practice to optimize the management of patients with a liver cancer [14].

There was a decreased risk of HCC with antiviral treatment that was based on clinical trial data using INF-based regimens, but recently, results of studies linking the use of DAA therapy with an increased risk of HCC present an interesting dilemma for clinicians [15].

In the current study, no development of $\mathrm{HCC}$ was noted in the studied group.

\section{Conclusion}

In the current study, 40 patients were treated with combined sofosbuvir $400 \mathrm{mg}$ and daclatasvir $60 \mathrm{mg}$ for 24 weeks. All patients received treatment at least 3 months following transplantation. Forty patients (100\%) have good response to treatment during treatment and during follow-up (SVR 12 was 100\%). No abnormal side effects to treatment were detected; also, no drug-drug interactions were noted during treatment. No HCC was detected during treatment and follow-up.

\section{Abbreviations}

HCV: Hepatitis C virus; SVR: Sustained virological response; DAAs: Directacting antivirals; RBV: Ribavirin; ALT: Alanine aminotransferase; AST: Aspartate aminotransferase; AFP: Alpha fetoprotein; HCV PCR: Hepatitis C virus polymerase chain reaction; CBC: Complete blood count; INR: International normalized ratio; IQR: Interquartile range; ESLD: End-stage liver disease; LT: Liver transplantation; PEG-INF: Pegylated interferon; CNI: Calcineurin inhibitors; HCC: Hepatocellular carcinoma; SPSS: Statistical Package for Social Sciences; EOT: End of treatment

\section{Acknowledgements}

Not applicable

\section{Authors' contributions}

OAA selected the patients from Ain Shams University Specialized Hospital and El Maadi Military Hospital. WAY made the design for the study. AME and NMT contributed to the follow-up of the patients and analysis and interpretation of the data. EMS contributed to the data collection. All authors provided critical feedback and helped shape the research, analysis, and manuscript. All authors have read and approved the final manuscript.

\section{Funding}

The funders had no role in the study design, data collection and analysis, decision to publish, or preparation of the manuscript.

\section{Availability of data and materials}

The authors confirm that the data supporting the finding of this study are available within the article.

\section{Ethics approval and consent to participate}

All data in this study were in accordance with the ethical standards of Ain Shams University Research Committee and with the 1964 Helsinki

Declaration and its later amendments.

Ethics committees reference number: 00017585.

Address: Faculty of Medicine, Ain Shams University, Cairo 11211, Egypt. Informed written consent was obtained from each participant before enrolment in the study.

\section{Consent for publication}

Informed consent to publish patient's data was signed by all participants prior to the beginning of the research.

\section{Competing interests}

The authors declare that they have no competing interests.

\section{Author details}

${ }^{1}$ Division of Gastroenterology and Hepatology, Department of Internal Medicine, Faculty of Medicine, Ain Shams University, Cairo, Egypt. ²Division of Nephrology, Department of Internal Medicine, Faculty of Medicine, Ain Shams University, Ramsis street 38, Abbasia, Cairo 11566, Egypt.

Received: 21 April 2020 Accepted: 14 September 2020

Published online: 24 September 2020

References

1. Jamieson N, McMaster P, O'Grady JG et al (2003) Evolution of liver transplantation in Europe: report of the European Liver Transplant Registry. Liver Transpl 9:1231-1243. https://doi.org/10.1016/j.lts.2003.09.018

2. Roche B, Samuel D (2007) Risk factors for hepatitis C recurrence after liver transplantation. J Viral Hepat 14(7):89-96. https://doi.org/10.1111/j.13652893.2007.00920.x

3. Berenguer M, Aguilera V, Prieto M (2008) Clinical benefits of antiviral therapy in patients with recurrent hepatitis $C$ following liver transplantation. Am J Transplant 8(3):679-687. https://doi.org/10.1111/j.1600-6143.2007.02126.x

4. Coilly A, Roche B, Dumortier J et al (2014) Safety and efficacy of protease inhibitors to treat hepatitis $C$ after liver transplantation: a multicenter experience. JHepatol 60:78-86

5. Lok AS (2013) HCV NS5A inhibitors in development. Clin Liver Dis 17(1):111121. https://doi.org/10.1016/j.cld.2012.09.006

6. Fontana R, Hughes E, Bifano M (2013) Sofosbuvir and daclatasvir combination therapy in a liver transplant recipient with severe recurrent cholestatic hepatitis C. American Journal of Transplantation 13:1601-1605 https://doi.org/10.1111/ajt.12209

7. Mallet V, Gilgenkrantz H, Serpaggi J et al (2008) Brief communication: The relationship of regression of cirrhosis to outcome in chronic hepatitis C. Ann. Intern. Med. chapter 149:399-403. https://doi.org/10.7326/0003-4819149-6-200809160-00006 
8. Poordad F, Schiff E, Vierling J et al (2016) Daclatasvir with sofosbuvir and ribavirin for hepatitis $C$ virus infection with advanced cirrhosis or post-liver transplantation recurrence. Hepatology 63(5):1493-1505 https://doi.org/10. 1002/hep.28446

9. Pellicelli AM, Montalbano M, Lionetti R et al (2014) Sofosbuvir plus daclatasvir for post-transplant recurrent hepatitis C: potent antiviral activity but no clinical benefit if treatment is given late. Digestive and Liver Disease 46:923-927

10. Leroy V, Dumortier J, Coilly A et al (2015) Efficacy of sofosbuvir and daclatasvir in patients with fibrosing cholestatic hepatitis $\mathrm{C}$ after liver transplantation. Clin Gastroenterol Hepatol 13(11):1993-2001.e1-2. https:// doi.org/10.1016/j.2015.05.030

11. Manns M, Reesink H, Berg T et al (2011) Rapid viral response of once-daily TMC435 plus pegylated interferon/ribavirin in hepatitis C genotype-1 patients: a randomized trial. AntiviralTherapy 16(7):1021-1033. https://doi. org/10.3851/IMP1894

12. Suraweera D, Sundaram V, Saab S (2016) Treatment of hepatitis C virus infection in liver transplant recipients. Gastroenterol. Hepatol 12(1):23-30

13. Price JC and Terrault NA (2015). Treatment of hepatitis $C$ in liver transplant patients: interferon OUT, direct antiviral combos IN. Liver Transpl; (4)21:423434. doi: https://doi.org/10.1002/lt.24080.

14. Trépo E, Nahon P, Bontempi $G$ et al (2014) Association between the PNPL A3 (rs738409 C>G) variant and hepatocellular carcinoma: evidence from a meta-analysis of individual participant data. Hepatology 59(6):2170-2177. https://doi.org/10.1002/hep.26767

15. Hirmatsu N, Oze T, Takehara T (2015) Suppression of hepatocellular carcinoma development in hepatitis C patients given interferon based antiviral therapy, Hepatol. Res 45(2):152-161. https://doi.org/10.1111/hepr.12393

\section{Publisher's Note}

Springer Nature remains neutral with regard to jurisdictional claims in published maps and institutional affiliations.

\section{Submit your manuscript to a SpringerOpen ${ }^{\circ}$ journal and benefit from:}

- Convenient online submission

- Rigorous peer review

- Open access: articles freely available online

- High visibility within the field

- Retaining the copyright to your article

Submit your next manuscript at $\boldsymbol{\nabla}$ springeropen.com 